\title{
Esclerose múltipla: implementação do processo de enfermagem
}

\section{Multiple sclerosis: implementationofthe nursing process}

\author{
Mariana Melo da Cruz Domingos ${ }^{1} \bullet$ Thatiane Monick de Souza Costa² • Bárbara Coeli Oliveira da Silva ${ }^{3}$ Francisca Marta \\ de Lima Costa Souza ${ }^{4}$ Cintia Capistrano Teixeira Rocha ${ }^{5} \bullet$ Richardson Augusto Rosendo da Silva ${ }^{6}$
}

\begin{abstract}
RESUMO
Objetivo: Elaborar, validar, implementar e avaliar um plano de cuidados de enfermagem a uma paciente com Esclerose Múltipla. Metodologia: Trata-se de um estudo de intervenção metodológica realizado em uma paciente de um Hospital Escola na capital do Nordeste do Brasil, no período de junho de 2014. Seguiram-se as seguintes etapas: elaboração dos Diagnósticos de Enfermagem; proposta inicial de resultados e intervenções, elaboração de um plano de cuidados e validação por especialista, implementação e avaliação do plano. Resultados: Elaborou-se, implementou e avaliou um plano de cuidados contendo cinco diagnósticos (mobilidade física prejudicada; dor crônica; sofrimento espiritual; memória prejudicada; eliminação urinária prejudicada) cinco metas, cinco intervenções e 21 atividades de enfermagem. Conclusões: Nota-se que a aplicabilidade do processo de enfermagem direciona as necessidades prioritárias à paciente, permitindo uma linguagem homogênea e cientifica entre os profissionais de enfermagem.
\end{abstract}

Palavras-chave: Esclerose Múltipla; Processos de Enfermagem; Cuidados de Enfermagem.

\begin{abstract}
Objective: To develop, validate, implement and evaluate a plan of nursing care to a patient with multiple sclerosis. Methodology: This is a methodological intervention study in a patient of a Teaching Hospital in the capital of Northeast Brazil, from June 2014. This was followed by the following steps: preparation of nursing diagnoses; Initial proposed outcomes and interventions, preparation of a plan of care and expert validation, implementation and plan evaluation. Results: We elaborated, implemented and evaluated a care plan with five diagnoses (impaired physical mobility, chronic pain, spiritual pain, impaired memory, impaired urinary elimination) five goals, five interventions and 21 nursing activities. Conclusions: It is clear that the application of the nursing process directs the priority needs the patient, allowing a homogeneous and scientific language among nursing professionals.
\end{abstract}

Keywords: Multiple Sclerosis; Nursing Process; Nursing Care.

${ }^{1}$ Enfermeira. Especialista em Enfermagem do Trabalho. Enfermeira Assistencial da Casa de Saúde São Lucas. Membro do Grupo de Pesquisa Práticas Assistenciais e Epidemiológicas em Saúde e Enfermagem - PAESE/UFRN. Natal/RN, Brasil. E-mail: marimcd@hotmail.com.

2 Enfermeira. Especialista em Enfermagem do Trabalho. Membro do Grupo de Pesquisa Práticas Assistenciais e Epidemiológicas em Saúde e Enfermagem - PAESE/UFRN. Natal/RN, Brasil.E-mail: thatianemonick@hotmail.com.

${ }^{3}$ Enfermeira. Especialista em Enfermagem em Cardiologia e Hemodinâmica. Mestranda do Programa de Pós-Graduação em Enfermagem da Universidade Federal do Rio Grande do Norte - UFRN. Professora Substituta do Curso de Graduação em Enfermagem da UFRN. Membro do Grupo de Pesquisa Práticas Assistenciais e Epidemiológicas em Saúde e Enfermagem - PAESE/UFRN. Natal/RN, Brasil. E-mail: barbaracoeli@outlook.com.

${ }^{4}$ Enfermeira. Mestre em Enfermagem pela Universidade Federal do Rio Grande do Norte - UFRN. Doutoranda do Programa de Pós-Graduação em Enfermagem da Universidade Federal do Rio Grande do Norte - UFRN. Enfermeira Assistencial da Maternidade Municipal Professor Leide Morais. Membro do Grupo de Pesquisa Práticas Assistenciais e Epidemiológicas em Saúde e Enfermagem - PAESE/UFRN. Natal/RN, Brasil. E-mail:enfermarta2001@yahoo.com.br.

${ }^{5}$ Enfermeira. Especialista em Enfermagem Clínica e Enfermagem do Trabalho. Mestranda do Programa de Pós-Graduação em Enfermagem da Universidade Federal do Rio Grande do Norte - UFRN. Enfermeira Assistencial da Casa de Saúde São Lucas. Professora Substituta da Faculdade de Ciências da Saúde do Trairi - FACISA/UFRN. Membro do Grupo de Pesquisa Práticas Assistenciais e Epidemiológicas em Saúde e Enfermagem - PAESE/UFRN. Natal/RN, Brasil. E-mail: cintiarocha_4@hotmail.com.

${ }^{6}$ Enfermeiro. Doutor em Ciências da Saúde pela Universidade Federal do Rio Grande do Norte - UFRN. Professor do Curso de Graduação e do Programa de Pós-Graduação (Mestrado Acadêmico e Doutorado) em Enfermagem da UFRN. Vice-líder do Grupo de Pesquisa Práticas Assistenciais e Epidemiológicas em Saúde e Enfermagem - PAESE/ UFRN. Natal/RN, Brasil. E-mail: rirosendo@hotmail.com. 


\section{INTRODUÇÃO}

A Esclerose Múltipla (EM) é caracterizada como uma doença autoimune, crônica, inflamatória, e desmielinizante que afetam o Sistema Nervoso Central (SNC). De etiologia ainda desconhecida, pode estar relacionada à predisposição genética e a fatores ambientais. Apresenta incidência maior em adultos jovens, do sexo feminino, de etnia branca, na faixa etária de 20 a 40 anos de idade ${ }^{1}$.

Os sinais e sintomas mais frequentes da EM são: neurite óptica, parestesias, nistagmo, diplopia, vertigens, disfunção vesical e sexual e uma diminuição da acuidade visual, levando à perda gradual da visão. A espasticidade, característica da doença, associa-se aos espasmos musculares espontâneos ou induzidos por movimentos ${ }^{2-3}$.

Assim, o enfermeiro possui um papel fundamental na assistência a pessoas com EM, seja na promoção, prevenção e tratamento, utilizando a Sistematização da Assistência de Enfermagem (SAE), a qual é um instrumento que auxilia na prestação do cuidado de forma cientifica e holística ${ }^{4}$.

Dentre os diversos meios sistemáticos tem-se o Processo de Enfermagem (PE) que ocorre em cinco etapas: coleta de dados ou histórico de enfermagem, identificação dos diagnósticos de enfermagem, planejamento de resultados e metas a cumprir, a implementação destas ações além de uma avaliação feita pelo profissional sobre o quadro do paciente ${ }^{5}$.

É necessário, ainda, a utilização dos sistemas de classificação, os quais auxiliam na descrição e comunicação das atividades da prática de enfermagem. Uma das classificações mais utilizadas na enfermagem é a NANDA Internacional (NANDA-I), para os diagnósticos de enfermagem ${ }^{6}$.

Para o desenvolvimento do estudo, buscou-se por produções cientificas dos últimos cinco anos relacionadas a elaboração, validação, implementação e avaliação de plano de cuidados a pessoas com EM, através dos descritores em ciências da saúde: Esclerose múltipla/ Multiple sclerosis; Processos de Enfermagem/ Nursing Process; Cuidados de Enfermagem/ Nursing Care, nas bases de dados informatizadas da Biblioteca Virtual em Saúde (BVS); Literatura Latino-Americana e do Caribe (Lilacs) e Literatura Internacional em Ciências da Saúde e Biomédica (Medline); SCOPUS e CINAHL. Dessa forma, percebeu-se uma escassez de estudos sobre a temática em questão.

Contudo, a relevância do estudo está em avaliar as necessidades prioritárias de pessoas com EM através de um plano de cuidados baseado nos preceitos da evidência científica. Diante dessa lacuna, justifica-se a realização da presente pesquisa. Nesse sentido, questiona-se: existem resultados frente à implementação do processo de enfermagem a uma paciente com Esclerose Múltipla? Assim, o estudo teve como objetivo elaborar, validar, implementar e avaliar um plano de cuidados de enfermagem a uma paciente com Esclerose Múltipla a partir da NANDA-INIC-NOC (NANDA-International - Nursing Interventions Classification - Nursing Outcomes Classification) ${ }^{6}$.

\section{METODOLOGIA}

Trata-se de estudo de intervenção metodológica, desenvolvido com uma paciente de diagnóstico médico com esclerose múltipla, presente na unidade de neurologia de um hospital escola, no Nordeste do Brasil.

Os dados foram coletados no período de junho de 2014, por meio de um roteiro de anamnese e exame físico que contemplava os aspectos sociodemográficos, clínicos e comportamentais. 0 instrumento foi submetido à validação do conteúdo e aparência por dez docentes que desenvolvem estudos na área da SAE. Posteriormente, as sugestões propostas foram contempladas no instrumento.

Em seguida, foi realizado um treinamento teórico e prático para padronizar a coleta de dados com dois alunos da pós-graduação a nível de Mestrado e Doutorado com carga horária de 12 horas semanais, desenvolvido por meio de aulas expositivas e dialogadas e discussões de casos clínicos com ênfase na abordagem às pessoas com Esclerose Múltipla. Após a etapa teórica do curso, realizouse uma atividade prática de simulação de exame físico em pares, com o intuito de capacitar o pesquisador e uniformizar a coleta de dados.

A trajetória metodológica seguiu as seguintes etapas: elaboração dos Diagnósticos de Enfermagem; proposta inicial de resultados e intervenções, elaboração de um plano de cuidados e validação por especialista, implementação e avaliação do plano.

A elaboração dos diagnósticos foi processual, realizada simultaneamente com a coleta de dados, buscando identificar as características definidoras e os fatores relacionados/de risco de acordo com a NANDA-I, versão 2012-2014. Ressalta-se que no processo de inferência diagnóstica, a história clínica da paciente foi avaliada pelos pesquisadores, a fim de possibilitar maior fidedignidade aos resultados obtidos.

Os resultados obtidos passaram por processo de revisão de forma pareada entre os autores, para assegurar um julgamento consensual, objetivando, assim, maior acurácia. Além disso, os resultados e as intervenções foram propostos pelos autores, para cada um dos diagnósticos mais frequentes, embasados na experiência clínica e nas sugestões das classificações da NIC e NOC. Consideram-se os principais diagnósticos formulados para mais de $50 \%$ dos participantes do estudo.

Após a construção da proposta do plano de cuidados, a mesma foi submetida a um processo de validação de conteúdo por especialistas da área, que concordaram em participar do estudoassinando o Termo de Consentimento Livre e Esclarecido (TCLE), cujo critério de seleção foi ter desenvolvido estudo publicado ou de conclusão de titulação (especialização, mestrado ou doutorado) relacionado aos diagnósticos de enfermagem em pacientes com afecções neurológicas ou ter orientações acadêmicas na área; e como critério de exclusão: informar no Currículo Lattes apenas o Trabalho de Conclusão do Curso de Graduação sobre a 
temática e a não atualização do currículo nos últimos 6 meses. Foram selecionados 16 enfermeiros especialistas.

Para o tratamento dos dados coletados, os instrumentos foram numerados e as variáveis foram codificadas e inseridas em banco de dados construído no programa Excel for Windows. Os dados foram analisados utilizando-se estatísticas descritivas. Para análise do grau de concordância entre o pesquisador e os especialistas, optou-se pelo índice de Kappa, analisado pelo programa Statistical Package for the Social Science (SPSS), versão 20.0. $O$ índice de Kappa foi definido como uma medida de associação para descrever e testar o grau de concordância, ou seja, confiabilidade e precisão de uma avaliação. Valores de Kappa > 0.80 são considerados como um bom nível de concordância. Após a proposta final, o plano foi implementado e avaliado pelos pesquisadores e realizadas as devidas modificações, conforme a necessidade.

O projeto foi aprovado pelo Comitê de Ética em Pesquisa da Universidade Federal do Rio Grande do Norte sob o CAAE No: 11984212.4.0000.553., processo No 267.215. Seguindo-se, assim, as normativas éticas nacionais e internacionais frente à pesquisa com seres humanos com assinatura no TCLE pela participante.

\section{RESULTADOS}

A partir das variáveis que compuseram os instrumentos, elencou-se os achados contemplados na anamnese e as características dispostas no exame físico constituindo, assim, o caso clinico da paciente.

\section{Apresentação do caso}

Paciente, sexo feminino, 49 anos, branca, natural do Interior do $R N$, solteira, com nível superior incompleto, diagnosticada com Esclerose Múltipla (EM) há 17 anos, nega alergia, diabetes e hipertensão. Mora com os pais, que cuidam dela, dedicando-lhe total assistência. Encontra-se internada no Hospital Universitário no Nordeste do Brasil decorrente de um quadro de recorrentes infecções urinarias. Ficou cega há aproximadamente 06 meses, tem um déficit de memória, como não lembra-se de acontecimentos passados; encontra-se acamada, pois apresenta tremores involuntários e instabilidade da marcha, relata apetite diminuído e necessita de ajuda para alimentar-se; psicologicamente instável, pois revolta-se com a sua condição de saúde, descrente de qualquer tipo de religião e esperança. Ao exame físico: consciente, orientada, hipocorada, anictérica, acianótica e desidratada. Com déficit visual bilateral. Ausência de linfonodos infartados. Pulsos periféricos palpáveis. Tórax simétrico e abdome plano. Ausculta pulmonar e cardíaca normais. Membros superiores $e$ inferiores sem edema, mas com discreta perda de massa muscular. Refere-se dor não localizada e sim generalizada, como também fadiga. Sinais Vitais: Temperatura $36,3^{\circ} \mathrm{C}$; Pulso: 87 bpm; Pressão Arterial: 130x80 mmhg; Frequência respiratória: 20 irpm.

\section{Plano de cuidados de enfermagem}

Após os dados coletados, foram identificados 14 diagnósticos de enfermagem, entretanto, apenas cinco diagnósticos estavam presentes em $50 \%$ dos entrevistados, sendo estes: Mobilidade física prejudicada; Dor crônica; Sofrimento espiritual; Memória prejudicada; Eliminação urinária prejudicada. Em seguida, estabeleceram-se as devidas metas e intervenções que tiveram o IC > 0.80 para que, assim, fosse implementado o plano de cuidados e avaliado os resultados.

Quadro 1: Teste de Relevância I - Aplicado à referência dos artigos. Belo Horizonte, 2014.

\section{Diagnóstico de Enfermagem: Mobilidade física prejudicada relacionado a dor e intolerância à atividade evidenciado por capacidade limitada para desempenhar as habilidades motoras finas e grossas.}

Meta: Movimento coordenado

Indicadores:

\begin{tabular}{|l|l|l|l|l|l|}
\hline Facilidade do movimento & & & 3 & & \\
\hline Controle do movimento & & & 3 & & \\
\hline Movimento na direção desejada & & & 3 & & \\
\hline Movimento no momento desejado & & & 3 & & \\
\hline
\end{tabular}

Intervenção: Terapia com exercício: controle muscular.

Atividades:

- Iniciar medidas de controle da dor antes do início do exercício/atividade;

- Explicar as razões da escolha do tipo de exercício e o protocolo ao paciente/ família;

- Consultar um fisioterapeuta para determinar a melhor posição para o paciente durante os exercícios e o número de repetições para cada padrão de movimento;

- Colaborar com o fisioterapeuta e terapeuta ocupacional no desenvolvimento e execução de programa de exercícios, conforme apropriado;

- Dar privacidade para que o paciente se exercite;

- Incorporar as atividades da vida diária (ADL) ao protocolo de exercícios, se apropriado;

A partir das intervenções estabelecidas e após uma semana do início da execução do plano de cuidados, foi realizada a primeira avaliação. Nesta, a paciente relatou sentir-se indisposta para realização das atividades diárias. O somatório dos indicadores apresentou um total de 7 pontos. $\mathrm{Na}$ avaliação seguinte, ao término da segunda semana, a paciente referiu melhora na capacidade de alimentar-se sozinha e aumento na disposição para realização das atividades diárias, obtendo 9 pontos no quadro dos indicadores. Ao final da terceira semana, última avaliação, a paciente relatou ter conseguido sentar e caminhar com dificuldade e ajuda do fisioterapeuta, atingindo um total de 12 pontos perante os indicadores (Quadro 1). 
Quadro 2: Plano de cuidados de enfermagem ao paciente com esclerose múltipla, conforme diagnóstico prioritário, Natal, Brasil, 2014.

\begin{tabular}{|l|l|l|l|}
\hline \multicolumn{3}{|l|}{ Diagnóstico de Enfermagem: Dor crônica relacionado à } \\
incapacidade física crônica evidenciado por relato verbal de \\
dor, expressão facial, depressão e alteração da capacidade de \\
continuar atividades prévias. \\
\hline Meta: Nível de dor \\
\hline Indicadores: \\
\hline Dor relatada & & 3 & \\
\hline Gemido e choro & & 4 \\
\hline Expressões faciais de dor & & & 4 \\
\hline Intervenção: Controle da dor & \multicolumn{3}{|l|}{} \\
\hline Atividades: \\
- Assegurar que o paciente receba cuidados precisos de \\
analgesia. \\
- Utilizar uma abordagem multidisciplinar para o controle da \\
dor quando adequado. \\
- Encorajar o paciente a conversar sobre sua experiência de \\
dor, conforme apropriado. \\
- Promover repouso/sono adequado para facilitar o alívio da dor. \\
- Instituir e modificar as medidas de controle da dor com base \\
na resposta do paciente. \\
- Usar medidas de controle da dor antes do seu agravamento. \\
- Determinar o impacto da experiência da dor na qualidade de \\
vida (sono, apetite, atividade, humor, relacionamentos) \\
\hline
\end{tabular}

No decorrer da implementação das atividades, realizou-se a primeira avaliação. Ao completar-se a primeira semana, a paciente relatou aumento do bem-estar após conversar sobre sua experiência de dor. A soma dos indicadores apresentou 5 pontos. Na segunda avaliação, a paciente referiu o alívio da dor, devido à diminuição da intensidade da mesma, obtendo 8 pontos no quadro dos indicadores. Na última avaliação, após a terceira semana, a paciente referiu melhora na qualidade de vida após a diminuição da dor, estabelecendo um total de 11 pontos na soma dos indicadores (Quadro 2).

Quadro 3: Plano de cuidados de enfermagem ao paciente com esclerose múltipla, conforme diagnóstico prioritário, Natal, Brasil, 2014.

\begin{tabular}{|c|c|c|}
\hline \multicolumn{3}{|c|}{$\begin{array}{l}\text { Diagnóstico de Enfermagem: Sofrimento espiritual relacionado } \\
\text { a doença crônica e morte evidenciado por raiva de um ser maior, } \\
\text { expressa sofrimento, falta de amor, falta de esperança e raiva. }\end{array}$} \\
\hline \multicolumn{3}{|c|}{ Meta: Enfrentamento } \\
\hline \multicolumn{3}{|l|}{ Indicadores: } \\
\hline $\begin{array}{l}\text { Identifica padrões eficazes de } \\
\text { enfrentamento }\end{array}$ & 4 & \\
\hline Relata redução do estresse & 4 & \\
\hline Verbaliza aceitação da situação & 4 & \\
\hline Relata aumento do conforto psicológico & 4 & \\
\hline \multicolumn{3}{|l|}{ Intervenção: Apoio Espiritual } \\
\hline \multicolumn{3}{|c|}{$\begin{array}{l}\text { Atividades: } \\
\text { - Estar aberto aos sentimentos da pessoa sobre doença e } \\
\text { morte. } \\
\text { - Ajudar o indivíduo a expressar e aliviar a raiva } \\
\text { adequadamente. } \\
\text { - Garantir ao indivíduo que o enfermeiro estará disponível } \\
\text { para apoia-lo em momentos de sofrimento. } \\
\text { - Estimular a participação em grupos de apoio. } \\
\text { - Estar disponível para ouvir os sentimentos individuais. }\end{array}$} \\
\hline
\end{tabular}

A primeira avaliação foi realizada uma semana antes do início da implementação do plano de cuidados. Nesta, a paciente relata não ter esperanças, demonstra estar revoltada com a situação em que se encontra e tem bastante medo de morrer. Sendo estabelecida a pontuação de 4 pontos em relação aos indicadores. Após ser orientada a executar métodos de relaxamento e meditação, dialogar com o enfermeiro sobre seu sofrimento e participar de grupos de apoio. Obteve 10 pontos no quadro de indicadores, referente à avaliação da segunda semana. No último dia de avaliação, ao findar a terceira semana, a paciente afirma melhora da autoestima e mudança do seu pensamento a respeito da doença e da morte, atingindo o total de 16 pontos ao somatório dos indicadores (Quadro 3).

Quadro 4: Plano de cuidados de enfermagem ao paciente com esclerose múltipla, conforme diagnóstico prioritário, Natal, Brasil, 2014.

Diagnóstico de Enfermagem: Memória prejudicada

relacionada a distúrbios neurológicos evidenciados por

incapacidade de recordar eventos, incapacidade de recordar informações reais e experiências de esquecimento.

\begin{tabular}{|c|c|c|}
\hline \multicolumn{3}{|l|}{ Meta: Cognição } \\
\hline \multicolumn{3}{|l|}{ Indicadores: } \\
\hline Orientação cognitiva & 3 & \\
\hline Demonstra memória recente & 3 & \\
\hline Demonstra memória remota & & 4 \\
\hline \multicolumn{3}{|c|}{ Intervenção: Treinamento da memória } \\
\hline \multicolumn{3}{|l|}{ Atividades: } \\
\hline \multicolumn{3}{|c|}{$\begin{array}{l}\text { - Proporcionar memória do reconhecimento de fotos e } \\
\text { gravuras, conforme apropriado. }\end{array}$} \\
\hline \multicolumn{3}{|c|}{$\begin{array}{l}\text { - Estimular a memória pela repetição do último pensamentc } \\
\text { que o paciente expressou, conforme apropriado. }\end{array}$} \\
\hline
\end{tabular}

Uma semana após o início da implementação das intervenções, foi realizado a primeira avaliação, na qual a paciente referiu esquecer algumas informações recentes e acontecimentos passados, sendo estabelecida a pontuação de 9 pontos. Na segunda avaliação, com o intervalo de mais uma semana, a paciente permanece com o déficit de memória, e com os 9 pontos no quesito dos indicadores. Concluída a terceira semana, a paciente afirma melhora para recordar-se de informações recentes, e ao somatório final dos indicadores obteve 10 pontos (Quadro 4).

Ao final da primeira semana, no decorrer da implementação das atividades, realizou-se a primeira avaliação, na qual a paciente relatou estar atenta aos sinais e sintomas de infecção urinária; ao somar os indicadores, apresentou 8 pontos. Na segunda avaliação, após uma semana da primeira, a paciente referiu o alívio da dor e queimação ao urinar, obtendo 10 pontos no quadro dos indicadores. Na última avaliação, ao término da terceira semana, a paciente referiu melhora na qualidade de vida devido à diminuição da frequência e urgência ao urinar, estabelecendo um total de 16 pontos na soma dos indicadores (Quadro 5). 
Quadro 5: Plano de cuidados de enfermagem ao paciente com esclerose múltipla, conforme diagnóstico prioritário, Natal, Brasil, 2014.

\begin{tabular}{|c|c|}
\hline $\begin{array}{l}\text { Diagnóstico de Enferm } \\
\text { relacionada a dano sen } \\
\text { urinário evidenciados } p \\
\text { urinária. }\end{array}$ & $\begin{array}{l}\text { dicada } \\
\text { hência }\end{array}$ \\
\hline Meta: Eliminação Uriná & \\
\hline Indicadores: & \\
\hline Dor ao urinar & 4 \\
\hline Queimação ao urinar & 4 \\
\hline Frequência ao urinar & 4 \\
\hline Incontinência urinária & 4 \\
\hline Intervenção: Controle d & \\
\hline $\begin{array}{l}\text { Atividades: } \\
\text { - Monitorar a eliminaçấ } \\
\text { consistência, odor, vol } \\
\text { - Ensinar ao paciente o } \\
\text { trato urinário. } \\
\text { - Anotar o horário da ú } \\
\text { apropriado. } \\
\text { - Orientar o paciente/fa } \\
\text { conforme apropriado. } \\
\text { - Orientar o paciente p } \\
\text { urinar, conforme apro }\end{array}$ & ão do \\
\hline
\end{tabular}

\section{DISCUSSÃO}

O diagnóstico de enfermagem Mobilidade física prejudicada $^{7}$ confirma-se no paciente da pesquisa por apresentar alguns sinais e sintomas motores como, tremores em membros superiores e inferiores, parestesia bilateral e hipotonia muscular. Estudos também apontam que essa caracterização sintomatológica decorre das lesões primarias nos plexos motores, acarretando um espasticidade e reflexos osteotendinosos acentuados. Cerca de $90 \%$ dos portadores de EM apresentam algum grau de espasticidade que provoca grande desconforto e limitações de movimento, afetando diretamente no movimento coordenado e restringindo ao leito ${ }^{8}$.

Por isso, torna-se necessária a terapia com exercícios de forma ativa ou passiva, para que possa obter controle muscular, mesmo que de forma parcial, esperando um movimento coordenado, sendo o exercício um excelente preditor de resultados. Pontua-se que o exercício aeróbico regular de intensidade moderada não induz exacerbações da EM e melhora a mobilidade funcional e a qualidade de vida?

O diagnóstico de dor crônica, este relacionado à incapacidade física crónica, decorrente da destruição progressiva das terminações nervosas e o aumento de substancias como as bradicinas, referindo-se a quadros de cefaleias e raquialgias ${ }^{7,10}$.

Nesse sentido, um estudo realizado com 85 pacientes diagnosticado com EM no hospital de Portugal revelou que a maioria dos pacientes apresentava cefaleias e as raquialgias (27\%), seguidos dos membros superiores $(20 \%)$.
Muitos relataram o quadro de dor como superficial (86\%) por oposição à profunda (14\%), ocasionando, assim, impacto na qualidade de vida, a dor interferiu significativamente com a atividade geral, o estado de ânimo, o trabalho, as relações com outras pessoas e a capacidade de diversão ${ }^{11}$.

Quanto ao diagnóstico de sofrimento espiritual 7 , sabe-se que alguns pacientes com EM tem uma maior prevalência em desenvolver quadros de depressão em comparação com a população em geral, variando entre 26 e $42 \%$, bem como quando comparado com pessoas com outras condições crônicas, corroborando com os achados do estudo, pois a paciente apresentava indícios de quadro depressivo, referindo-se a sua condição de saúde. Assim, a depressão contribui para o processamento mais lento das informações, por parte dos pacientes, decorrente da redução da perfusão cerebral, o que acaba por comprometer aspectos cognitivos, dentre eles a atenção, aprendizagem, habilidades visuoespaciais, memória e a velocidade de processamento. Provoca, também, sofrimento psíquico e diminui a adesão ao tratamento, aumentando, por conseguinte, o risco de suicídio em pacientes com EM ${ }^{12}$.

O diagnóstico de enfermagem Memória prejudicada, esteve relacionado aos distúrbios neurológicos, em concordância ao déficit de memória presente na paciente em questão, devido à desmielinização dos neurônios ${ }^{7}$. Corroborando com os achados, estudo realizado com 28 pacientes atendidos em um ambulatório revelou que $89,2 \%$ tinham perda na memória de trabalho e $85,7 \%$, na memória de curto prazo. A deterioração cognitiva afeta a velocidade de processamento de informações e a memória, e, consequentemente, afeta as atividades de vida diária, o autocuidado e reduz a participação nas atividades sociais, aumentando a dependência do cuidado por outras pessoas $^{3,9-10}$.

Os pacientes com EM apresentam dificuldades de concentração e memória. Para possibilitar a melhoria da qualidade de vida, torna-se importante realizar, como rotina, uma avaliação precisa das funções cognitivas e atividades de reabilitação neuropsicológica como a realização de tarefas que exigem velocidade de memória e processamento de forma conjunta entre os profissionais de saúde ${ }^{13}$.

Relacionado ao DE, eliminação urinária prejudicada ${ }^{7}$, 0 paciente com EM apresenta uma deterioração no sistema vesico-motor interferindo diretamente na sensibilidade do trato urinário. Pois, em um estudo, verificou-se que o comportamento da incontinência urinária na esclerose múltipla e seu impacto na qualidade de vida dos pacientes acarretam danos tanto de cunho fisiológico como psicológico. Destacando que o processo de eliminação urinaria tornase um fator para o desenvolvimento de enfermidades secundarias como a úlcera por pressão, associado à incontinência fecal ${ }^{14-15}$. Diante disto, as intervenções de enfermagem devem ter como resultado uma melhora na eliminação urinaria, proporcionado uma qualidade de vida satisfatória, vislumbrado na paciente do estudo. 


\section{CONCLUSÕES}

Após a análise dos dados, nota-se que a implementação do processo de enfermagem à paciente com esclerose múltipla proporcionou um cuidado direcionado para as reais necessidades da cliente, fornecendo meios para propor as intervenções e alcançar os resultados. Contudo, é notório que o desenvolvimento do estudo se torna primordial para a composição dos saberes científicos, tanto do âmbito acadêmico como no assistencial. É perceptível, ainda, uma deficiência de produção nesse segmento diante das pessoas acometidas pela enfermidade.

Os diagnósticos de enfermagem prioritários a paciente foram Mobilidade física prejudicada; Dor crônica; Sofrimento espiritual; Memória prejudicada; Eliminação urinária prejudicada. 0 estudo limita-se à realidade de um município do Nordeste do país, mas acredita-se que esse trabalho possa incentivar novas pesquisas sobre a implementação do processo de enfermagem a pacientes com esclerose múltipla.

Espera-se que os resultados contribuam para os avanços e incorporação desta linguagem universal na descrição da prática profissional, em prol da melhoria da qualidade da assistência de enfermagem no cenário nacional

\section{REFERÊNCIAS}

1. Feinstein A. The neuropsychiat ry of multiple sclerosis. Can J Psychiatry 2013; 49(3): 157-63.

2. Morrison JD, Stuifbergen AK. Outcome expectations and physical activity in persons with longstanding multiple sclerosis. J Neurosci Nurs. 2014; 46(3): 171-79.

3. Freitas JOF, Aguiar CRRA. Avaliação das Funções Cognitivas de Atenção, Memória e Percepção em Pacientes com Esclerose Múltipla. Rev Psic ReflCrí.2012; 3(25): 457-66.

4. Corso NAA, Gondim APS, Almeida PCR, Albuquerque MGF. Sistematização da assistência de enfermagem para acompanhamento ambulatorial de pacientes com esclerose múltipla. Rev esc enferm USP. 2013; 47(3): 750-5.

5. Cavalcante I AMRZ, Brunoril EHFR, Lopes C T, Silva ABV, Herdman TH. Nursing diagnoses and interventions for a child after cardiac surgery in an intensive care unit. Rev Bras Enferm. 2015; 68(1): 155-60.

6. Park H. Identifying Core NANDA-I Nursing Diagnoses, NIC Interventions, NOC Outcomes, and NNN Linkages for Heart Failure. Int J Nurs Knowl. 2014; 25(1): 30-8.

7. Nanda Intenacional. Diagnósticos de Enfermagem da NANDA: definições e classificações 2010-2012. Porto Alegre: Artmed; 2013.

8. Strickland K, Baguley $\mathrm{F}$. The role of the community nurse in care provision for people with multiple sclerosis. $\mathrm{Br} J$ Community Nurs. 2015; 20(1): 6-10.

9. Morrison JD, Stuifbergen AK. Outcome expectations and physical activity in persons with longstanding multiple sclerosis. J Neurosci Nurs. 2014; 46(3): 171-9.

10. Almasalha F, Xu D, Keenan GM, Khokhar A, Yao Y,Chen J, et al. Data mining nursing care plans of endof- life patients: a study to improve healthcare decision making. Int J Nurs Knowl.2013; 24(1): 15-24.

11. Seixas D, Galhardo V, Sá MJ, Guimarães J, Lima D. Dor na esclerose múltipla: Caracterização de uma População Portuguesa de 85 Doentes. Acta Med Port. 2009; 22(3): 23340.

12. Askari F, Ghajarzadeh M, Mohammadifar M, Azimi A, Sahraian MA, Owji M. Anxiety in patients with multiple sclerosis: association with disability, depression, disease type and sex. Acta Med Iran. 2014; 52(12): 889-92.

13. Ben-Zacharia AB. Therapeutics for multiple sclerosis symptoms. Mt Sinai J Med. 2011; 78(2): 176-91.

14. Morrison JD, Stuifbergen AK. Outcome expectations and physical activity in persons with longstanding multiple sclerosis. J Neurosci Nurs. 2014; 46(3): 171-9.

15. Pavan K, Miguez PB, Marangoni BEM, Tilbery CP, Lianza S. Comportamento da incontinência urinária em pacientes com esclerose múltipla e a sua influência na qualidade de vida. Rev Med Reabil. 2010; 29(1): 1-5. 